\title{
Hidradenite Supurativa: Compreender para Tratar
}

\section{Hidradenitis Suppurativa: Understanding to Treat}

Urmal I' , Ortins-Pina A $^{2}$, Correia M³ $^{3}$, Silva JM $M^{2,3,4}$

\section{RESUMO}

A hidradenite supurativa é uma doença inflamatória cutânea, de caráter crónico e altamente debilitante, causada por oclusão folicular e consequente inflamação pilossebácea, manifestando-se através de nódulos, abcessos, fibrose e trajetos fistulosos. O profundo impacto que a hidradenite supurativa tem na qualidade de vida dos doentes levou a que, na última década, se tenha assistido a um crescente interesse da comunidade médica e científica. Este trabalho tem por objetivo a abordagem global da hidradenite supurativa tendo, para tal, sido conduzida uma pesquisa na base de dados bibliográfica PubMed/MEDLINE. Foram incluídos artigos originais, artigos de revisão e meta-análises, escritos apenas em língua inglesa. Da pesquisa efetuada, concluímos que existem questões fundamentais que carecem de esclarecimento, essencialmente no que diz respeito à definição dos mecanismos fisiopatológicos e fatores de risco associados, história natural e abordagem terapêutica da hidradenite supurativa.

PALAVRAS-CHAVE: Doenças Bacterianas da Pele; Hidradenite Supurativa/diagnóstico; Hidradenite Supurativa/ epidemiologia; Hidradenite Supurativa/fisiopatologia; Hidradenite Supurativa/tratamento

\section{ABSTRACT}

Hidradenitis suppurativa is an inflammatory, chronic, debilitating follicular skin disease, manifested as painful nodules, abcesses, scarring and sinus tracts. Despite its significant impact on patients' quality of life, only in the last decade hidradenitis suppurativa caught the attention of the scientific community.

This article provides a comprehensive review on hidradenitis suppurativa, based on articles sourced from the bibliographic database PubMed/MEDLINE. We included original articles, review articles and meta-analysis, written in english. From our research we concluded that further investigation is necessary in order to better define risk factors, pathological mechanisms, natural history and treatment of hidradenitis suppurativa.

KEYWORDS: Hidradenitis Suppurativa/diagnosis; Hidradenitis Suppurativa/epidemiology; Hidradenitis Suppurativa/physiopathology; Hidradenitis Suppurativa/therapy

1. Departamento de Ciências Biomédicas e Medicina, Universidade do Algarve, Faro, Portugal. 2. Clínica Universitária de Dermatologia, Faculdade de Medicina, Universidade de Lisboa, Lisboa, Portugal. 3. Centro de Dermatologia, Hospital CUF Descobertas, Lisboa, Portugal. 4. Clínica CUF Alvalade, Lisboa, Portugal.

Recebido: 12/06/2016 - Aceite: 02/09/2016 


\section{INTRODUÇÃO}

A hidradenite supurativa (HS), também conhecida como acne inversa ou doença de Verneuil, é uma doença inflamatória cutânea que resulta da oclusão crónica do folículo pilossebáceo, tendo um forte impacto na qualidade de vida dos indivíduos afetados. Caracteriza-se por uma inflamação folicular que afeta, essencialmente, as regiões axilar, inguinal, genital, inframamária e perineal, manifestando-se inicialmente por nódulos dolorosos recorrentes, progredindo mais tarde para formação de abcessos, fibrose, sinus e trajetos fistulosos. ${ }^{1,2}$ Considera-se que a oclusão folicular seja o mecanismo primário na fisiopatologia da hidradenite supurativa, resultando na acumulação de resíduos celulares e, consequentemente, formação de quistos. ${ }^{3}$ Existem vários fatores de risco identificados, nomeadamente fatores genéticos, hormonais, tabagismo e obesidade, embora o seu papel na fisiopatologia da HS ainda não esteja estabelecido. ${ }^{4} \mathrm{~A}$ abordagem terapêutica é dirigida para a prevenção de novas lesões e consequente progressão da doença, mas também para a eliminação das lesões pré-existentes. Esta inclui medidas não-farmacológicas, essencialmente através da promoção de um estilo de vida saudável, agentes tópicos, retinoides, anti-inflamatórios, antibióticos, agentes biológicos e, finalmente, intervenção cirúrgica. ${ }^{5}$

\section{EPIDEMIOLOGIA}

Estima-se que a hidradenite supurativa afete entre $1 \mathrm{e}$ $4 \%$ da população europeia, predominantemente mulheres, num ratio de 3:1. ${ }^{4}$ Apesar de a maioria dos estudos epidemiológicos terem sido realizados na Europa e Estados Unidos, não existem dados disponíveis em relação à população portuguesa. As suas manifestações clínicas da HS surgem habitualmente após a puberdade, tipicamente no início da segunda década de vida, permanecendo ativa até à quarta década de vida. ${ }^{6-8}$ Esta encontra-se associada a diversas doenças genéticas e autoimunes, nomeadamente doença inflamatória intestinal, espondiloartropatias, pioderma gangrenoso e síndrome de Down, entre outros. ${ }^{9-11}$

\section{FISIOPATOLOGIA}

Os mecanismos fisiopatológicos responsáveis pela hidradenite supurativa ainda não se encontram devidamente explicados. Várias fontes sugerem que seja uma doença multifatorial, cujo evento desencadeante é a oclusão folicular, resultando na acumulação de resíduos celulares e formação de quistos, que evoluem posteriormente para destruição do folículo piloso e originam fibrose, sinus e trajetos fistulosos. ${ }^{3}$ Apesar de o seu papel na fisiopatologia da HS ainda não estar bem estabelecido, existem vários fatores de risco identificados, nomeadamente fatores genéticos, hormonais, tabagismo e obesidade. Vários estudos demonstraram que 30-40\% dos doentes apresenta história familiar de HS, tendo sido identificadas, nas últimas décadas, várias mutações envolvendo genes reguladores da diferenciação celular da epiderme e do folículo piloso. ${ }^{12-14}$ Do mesmo modo, e apesar de não ser uma doença de etiologia infeciosa, os agentes bacterianos parecem desempenhar um papel relevante na fisiopatologia da hidradenite supurativa, sendo potencialmente responsáveis pela sobreinfeção e consequente agravamento das lesões. ${ }^{15}$ Globalmente, de entre todos os fatores de risco associados ao desenvolvimento de HS, o tabaco é aquele que apresenta uma maior relação de causalidade e maior consistência entre os estudos existentes, sendo os fumadores afetados mais frequentemente e com maior gravidade do que os não-fumadores. ${ }^{4} \mathrm{Na}$ base desta relação pode estar a ativação de fatores pro-inflamatórios, aumento da suscetibilidade folicular a invasão bacteriana e agravamento da sobreinfeção por bactérias como Staphylococcus aureus. Também o excesso de peso pode agravar a hidradenite supurativa através do aumento da fricção e do stress mecânico nas pregas cutâneas, desencadeando uma cascata promotora da oclusão folicular. Adicionalmente, a obesidade caracteriza-se por um estado pró-inflamatório, obtendo-se um efeito de sinergia com os mecanismos fisiopatológicos de base da HS.3,16-21

Adicionalmente, vários fatores hormonais podem contribuir para a oclusão folicular na HS, com base no facto de, em algumas mulheres, estarem descritas exacerbações da hidradenite supurativa durante o período pré-menstrual, bem como após o início de um contracetivo oral combinado. Apesar de esta relação ainda não estar bem estabelecida, tem vindo a verificar-se uma resposta positiva à terapêutica com agentes antiandrogénicos, cujo mecanismo na HS permanece por explicar.?

\section{APRESENTAÇÃO CLÍNICA}

A hidradenite supurativa caracteriza-se, inicialmente, por nódulos inflamatórios profundos (Fig. 1.1) dolorosos, que se vão tornando progressivamente mais superficiais e confluentes (Figs. 1.2 e 1.3). Afeta maioritariamente as regiões de distribuição das glândulas apócrinas - axilar (Figs. 1 e 2.1), inframamária (Fig. 2.2), inguinal (Fig. 2.4), genital e perianal. Os nódulos surgem frequentemente no período pré-menstrual, podendo durar entre 7 a 10 dias, caso não haja qualquer intervenção. A sua rotura pode ocorrer de forma espontânea, com drenagem de conteúdo purulento, sendo alvo de um processo de fi- 
brose à medida que cicatriza, culminando na formação de sinus e trajetos fistulosos. Simultaneamente surgem novas lesões nas regiões adjacentes, que podem permanecer intactas, evoluindo posteriormente para massas inflamatórias com potencial para sobreinfeção bacteriana e formação de abcessos, resultando numa inflamação crónica com extensas áreas de fibrose subcutânea (Fig. 2). ${ }^{22} \bigcirc$ diagnóstico diferencial da hidradenite supurativa inclui furunculose, celulite, foliculite, quisto dermoide, quisto epidermoide inflamado, doença de Crohn com envolvimento cutâneo, lesões neoplásicas primárias ou secundárias, sinus pilonidal, fístulas anais, abcessos perirretais, linfogranuloma venéreo e, embora mais raras, actinomicose e tuberculose cutâneas?

A ferramenta mais amplamente utilizada na classificação da HS é a classificação de Hurley (Tabela 1), desenvolvida em 1989, com base nas características das lesões. ${ }^{23}$ Atualmente são vários os métodos em estudo para avaliação da resposta à terapêutica, nomeadamente as escalas Physician Global Assessment (PGA), Hidradenitis Suppurativa Severity Index (HSSI) e Hidradenitis Suppurativa Clinical Response (HiSCR).

\section{TABELA 1. Classificação de Hurley.}

\begin{tabular}{l|l} 
Classificação de Hurley \\
$\begin{array}{l}\text { Estádio I } \\
\text { (ligeiro) }\end{array}$ & $\begin{array}{l}\text { Presença de nódulos inflamatórios e } \\
\text { abcessos, mas sem fibrose ou cicatrizes }\end{array}$ \\
\hline $\begin{array}{l}\text { Estádio II } \\
\text { (moderado) }\end{array}$ & $\begin{array}{l}\text { Presença de nódulos inflamatórios e } \\
\text { abcessos com fibrose associada e cicatrizes, } \\
\text { embora separadas por regiões íntegras, sem } \\
\text { lesões }\end{array}$ \\
\hline $\begin{array}{l}\text { Estádio III } \\
\text { (grave) }\end{array}$ & $\begin{array}{l}\text { Envolvimento difuso, com processos } \\
\text { fibróticos extensos e múltiplos sinus }\end{array}$ \\
\hline
\end{tabular}

(Adaptado de Hurley H. Axillary hyperhidrosis, apocrine bromhidrosis, hidradenitis suppurativa, and familial benign pemphigus: surgical approach. In: Roenigh RR, editor. Dermatologic Surgery. New York: Marcel Dekker;1989.p. 729-39).

\section{TRATAMENTO E PROGNÓSTICO}

$\bigcirc$ tratamento da hidradenite supurativa apresenta-se como um verdadeiro desafio, não só pelas inúmeras opções terapêuticas disponíveis, mas também pela escassez de evidência científica que suporte uma decisão clínica esclarecida. ${ }^{5,24}$ A sua abordagem deve dirigir-se, simultaneamente, para a prevenção de novas lesões e consequente progressão da doença, bem como para a remoção de lesões pré-existentes de modo a diminuir a formação de cicatrizes. Existem várias medidas passíveis de ser tomadas com o objetivo de reduzir a frequência das exacerbações e impedir a progressão da doença, limitando assim o aparecimento de novas lesões. A cessação tabágica é fundamental, aliada a uma alimentação equilibrada e à prática de exercício físico, bem como a prevenção de eventos traumáticos que possam potenciar a rotura dos nódulos inflamatórios.

\section{TERAPÊUTICA FARMACOLÓGICA}

Agentes tópicos - $\mathrm{O}$ tratamento de primeira linha da HS ligeira é a aplicação de clindamicina tópica duas vezes por dia durante dois a três meses, sendo este o único antibiótico tópico com evidência científica para utilização na HS. A clindamicina atua no tratamento das lesões superficiais sobreinfetadas, tendo também um papel profilático, ao prevenir as exacerbações causadas por infeções secundárias. Para além da antibioterapia, existem outros fármacos utilizados topicamente no tratamento da HS, como é o caso do resorcinol, amplamente utilizado na acne vulgaris pelas suas propriedades queratolíticas, anti-inflamatórias e antifúngicas. $\bigcirc$ seu papel na terapêutica da HS relaciona-se com a rotura precoce dos nódulos inflamatórios, levando a uma diminuição da dor a eles associada, bem como a uma redução da duração dos surtos. Apesar de não existirem estudos que comprovem o seu benefício, pensa-se que o ácido azelaico possa também ser utilizado no tratamento da hidradenite supurativa, pela sua ação anti-proliferativa. ${ }^{5,25}$

Antibioterapia - A antibioterapia sistémica está habitualmente reservada para o estádio II ou casos refratários à terapêutica. Embora a monoterapia com doxiciclina, minociclina ou clindamicina seja comum, a associação entre clindamicina e rifampicina é das mais utilizadas e melhor estudadas no contexto clínico da HS. Um estudo observacional de Gener et al, acompanhou durante 10 semanas 116 doentes tratados com clindamicina 300 mg bid e rifampicina 600 mg id, com evidência de melhoria através da redução do score de Sartorius. Pensa-se que a sua eficácia possa estar relacionada, em parte, com as propriedades anti-inflamatórias e imunomoduladoras das tetraciclinas, nomeadamente na supressão de linfócitos, neutrófilos e histiócitos, presentes em grande quantidade nas lesões da HS. 5,26,27

Anti-inflamatórios - A terapêutica anti-inflamatória da HS inclui administração de corticoides intra-lesionais, podendo ser utilizados enquanto monoterapia ou como adjuvante a outros fármacos. $\bigcirc$ agente mais frequente é o acetonido de triamcinolona, na dose de 5-10 mg/mL. Quando eficaz, verifica-se uma resposta entre 48-72 h após a administração, com redução significativa da inflamação das lesões, particularmente quando são localizadas e de pequenas dimensões. A corticoterapia sistémica é também uma opção para as exacerbações, não só devido ao seu caráter anti-inflamatório e imunossupressor, mas também pelos seus efeitos anti-proliferativos e vasoconstritores. $^{28}$

Retinoides orais - A isotretinoína tópica e sistémica é atualmente utilizada em larga escala, e com elevada eficácia terapêutica, no tratamento da acne vulgaris. Neste sentido, e com base nos seus efeitos anti-queratinizan- 

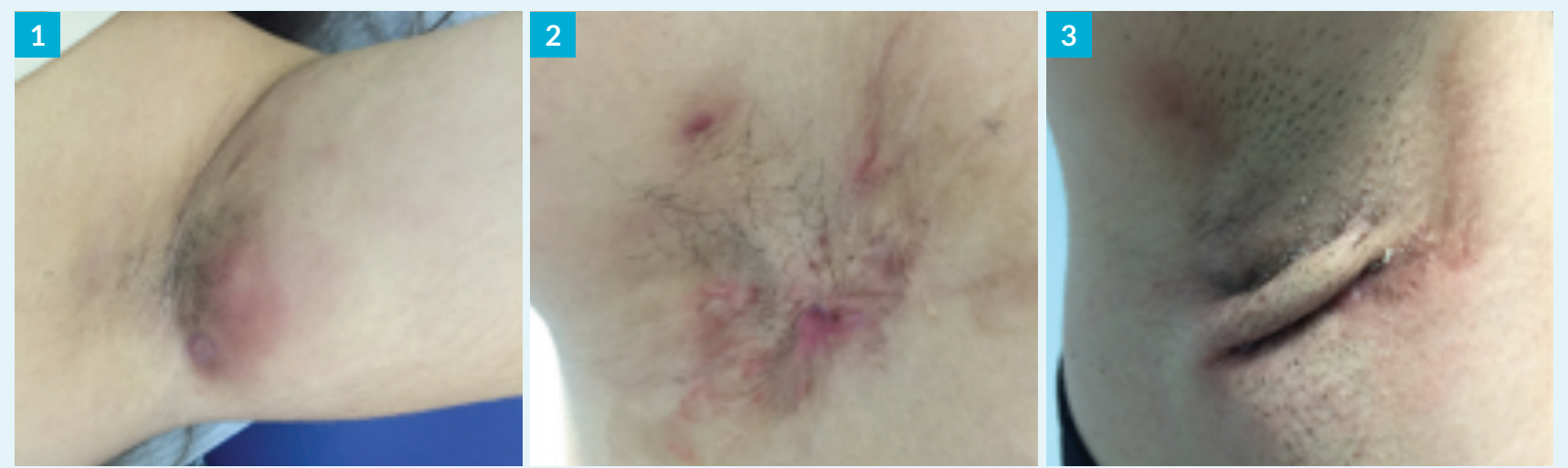

FIGURA 1. (1) Nódulo inflamatório na axila de doente com hidradenite supurativa no estádio de Hurley I; (2) Nódulos inflamatórios associados a fibrose, separados por pele íntegra, na axila de doente com hidradenite supurativa no estádio de Hurley II; (3) Nódulos inflamatórios e abcessos associados a cicatriz, separados por pele íntegra, na axila de doente com hidradenite supurativa no estádio de Hurley II.
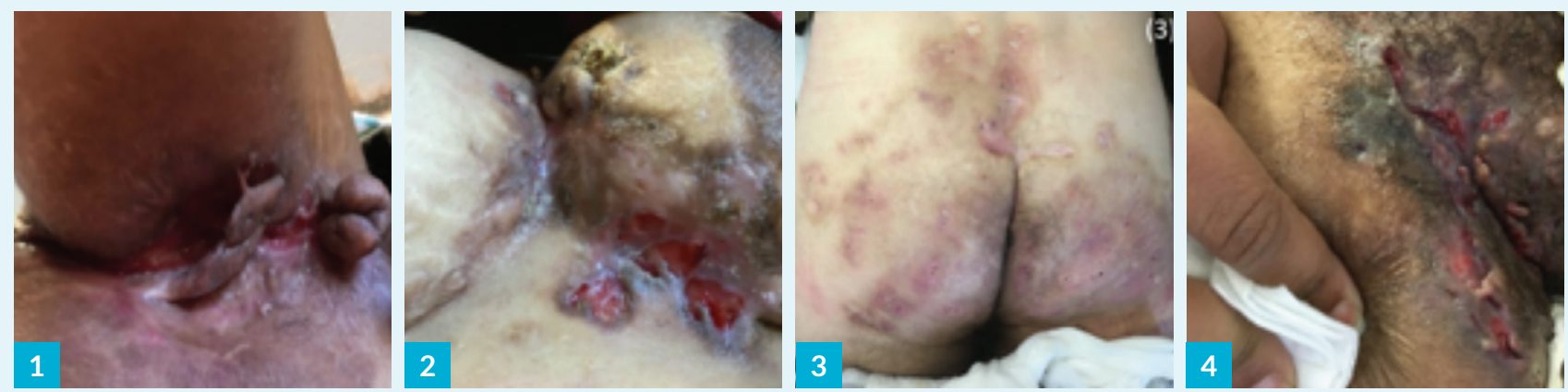

FIGURA 2. Envolvimento difuso das axilas (1), prega inframamária (2), região sagrada e glútea (3) e inguinal (4) com nódulos inflamatórios, abcessos, processos fibróticos extensos e múltiplas fístulas/orifícios de drenagem espontânea em doentes com HS no estádio de Hurley III.

tes e anti-inflamatórios, a isotretinoína tem vindo a ser utilizada nos doentes com HS. No entanto, a evidência científica disponível é limitada, pelo que se encontram em estudo outros agentes retinoides. A acitretina é um retinoide de segunda geração, aprovado para o tratamento da psoríase, com resultados promissores no tratamento da HS refratária. ${ }^{29-31}$

Agentes biológicos - Os agentes biológicos ganharam relevo nas últimas décadas no tratamento de doenças inflamatórias como a artrite reumatoide e psoríase. Os resultados positivos obtidos ao longo dos anos levaram à sua extrapolação para o tratamento de outras doenças de caráter inflamatório, como é o caso da hidradenite supurativa. $^{32}$

O adalimumab é um anticorpo monoclonal com atividade específica anti-TNF $\alpha$, tendo sido identificado como um dos fármacos mais eficazes na terapêutica da HS e sendo atualmente o agente biológico mais estudado. Neste sentido, e com base na evidência científica disponível, é também o mais utilizado no tratamento da doença avançada. ${ }^{33-36} \mathrm{O}$ infliximab é, tal como o adalimumab, um anticorpo monoclonal utilizado em grande escala no tratamento da HS. A grande desvantagem da sua utilização prende-se com a necessidade de administração intravenosa durante 1-2 h e vigilância do doente na hora seguinte à administração devido à possibilidade de reações ad- versas (flushing, cefaleias, náuseas, dispneia e alterações cutâneas no local da injeção). ${ }^{33,36,37}$ Do mesmo modo, o etanercept é um antagonista do TNF- $\alpha$ que tem vindo a apresentar resultados positivos em vários estudos, embora a sua interpretação deva ser cuidadosa atendendo ao reduzido número de doentes envolvidos. 36,38,39 Para além dos agentes mencionados, encontram-se em estudo outros fármacos, como é o caso do ustekinumab, utilizado habitualmente no tratamento da psoríase. Este tem vindo a ser utilizado em alguns doentes refratários à terapêutica com adalimumab, apresentando resultados promissores. ${ }^{40,41}$ Também o anakinra tem vindo a ser considerado como uma possível alternativa aos agentes biológicos já existentes. À semelhança do etanercept e do ustekinumab, os estudos realizados apresentaram resultados positivos, embora maior evidência científica seja necessária para suportar a utilização destes fármacos na HS. $36,42,43$

Outros - Pelos seus efeitos anti-androgénicos, nomeadamente na inibição na $5 \boldsymbol{\alpha}$-redutase tipo II, a finasterida pode atuar na redução da inflamação a nível do folículo piloso. No entanto, a sua utilização está contraindicada em mulheres em idade fértil e grávidas, devido ao risco de efeitos adversos para o feto.

A dapsona é um fármaco com propriedades antibacterianas e anti-inflamatórias, eficaz no tratamento de der- 


\section{PROGRESSÃO DA DOENÇA}

\section{EXCISÃO LOCAL, \\ DESBRIDAMENTO \\ TERAPÊUTICA \\ COM LASER}

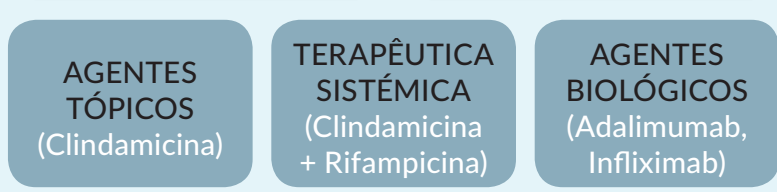

TERAPÊUTICA ADJUVANTE

Tratamento da dor

Tratamento das infeções secundárias

Perda de peso, exercício físico e cessação tabágica

FIGURA 3. Abordagem terapêutica da hidradenite supurativa (Adaptado de Zouboulis CC, et al. European S1 Guideline for the treatment of hidradenitis suppurativa/acne inversa. J Eur Acad Dermatol Venereol. 2015;29:619-44). ${ }^{5}$

matoses com infiltrados neutrofílicos proeminentes ou deposição de complexos imunes, que pode ter alguma relevância na fase inicial da HS, justamente mediada por neutrófilos. Também a metformina pode ter um papel relevante no tratamento da hidradenite supurativa, por se considerar que pode existir uma associação entre HS e insulinorresistência, ainda por esclarecer. ${ }^{5}$

\section{ABORDAGEM CIRÚRGICA}

A abordagem cirúrgica parece ser a única opção terapêutica efetivamente curativa, existindo, no entanto, vários procedimentos e técnicas passíveis de ser utilizados, desde a excisão local ou completa da área afetada, incisão e drenagem ou desbridamento, até às técnicas menos invasivas, como é o caso do laser de $\mathrm{CO}_{2}$. Este último veio diminuir a necessidade de utilização de enxertos, por permitir o tratamento de áreas extensas. $\mathrm{O}$ laser de dióxido de carbono atua através da vaporização das lesões, atingindo os planos profundos do tecido celular subcutâneo e fáscias musculares, e sendo por isso útil no tratamento de lesões infetadas secundariamente. Nos vários estudos realizados, os resultados obtidos evidenciaram uma melhoria significativa em todos os doentes tratados, com variações dependendo das áreas tratadas. ${ }^{24,44,45}$

As taxas de recorrência variam bastante entre técnicas e não podem atualmente ser comparadas visto que os estudos disponíveis utilizaram diferentes períodos de follow-up, bem como diferentes definições de recorrência. No que concerne ao tipo de cicatrização também não existe consenso, podendo ser utilizados enxertos, encerramento primário com sutura ou cicatrização por segunda intenção.

\section{CONCLUSÃO E PERSPETIVAS FUTURAS}

A hidradenite supurativa, com o seu profundo impacto na qualidade de vida dos doentes e uma obstinada refratariedade às tentativas para o minimizar, representa um exigente desafio para os médicos, quer do ponto de vista técnico-científico, quer do ponto de vista da relação médico-doente. No entanto, tem vindo a verificar-se um aumento do interesse por esta temática na última década, com um crescente número de estudos em curso. De modo a permitir uma abordagem terapêutica dirigida, existem questões fundamentais que devem ser esclarecidas num futuro próximo, essencialmente no que diz respeito à fisiopatologia da hidradenite supurativa e fatores de risco a ela associados.

CONFLITOS DE INTERESSE: Os autores declaram a inexistência de conflitos de interesse na realização do presente trabalho.

FONTES DE FINANCIAMENTO: Não existiram fontes externas de financiamento para a realização deste artigo.

\section{REFERÊNCIAS}

1. Dufour DN, Emtestam L, Jemec GB. Hidradenitis suppurativa: a common and burdensome, yet under-recognised, inflammatory skin disease. Postgrad Med J. 2014;90:216-21.

2. Jemec GB. Clinical practice. Hidradenitis suppurativa. N Eng J Med. 2012;366:158-64.

3. Prens E, Deckers I. Pathophysiology of hidradenitis suppurativa: An Update. J Am Acad Dermatol. 2015;73:S8-11.

4. Canoui-Poitrine F, Revuz JE, Wolkenstein P, Viallette C, Gabison G, Pouget F, et al. Clinical characteristics of a series of 302 French patients with hidradenitis suppurativa, with an analysis of factors associated with disease severity. J Am Acad Dermatol. 2009;61:51-7.

5. Zouboulis CC, Desai N, Emtestam L, Hunger RE, Ioannides D, Juhász I, et al. European S1 Guideline for the treatment of hidradenitis suppurativa/acne inversa. J Eur Acad Dermatol Venereol. 2015;29:619-44

6. Vazquez BG, Alikhan A, Weaver AL, Wetter DA, Davis MD. Incidence of hidradenitis suppurativa and associated factors: a population-based study of Olmsted County, Minnesota. J Invest Dermal. 2013;133:97-103.

7. Jemec GB, Kimball BA. Hidradenitis suppurativa: Epidemiology and scope of the problem. J Am Acad Dermatol. 2015;73:S4-7

8. McMillan K. Hidradenitis suppurativa: number of diagnosed patients, demographic characteristics, and treatment patterns in the United States. Am J Epidemiol. 2014;179:1477-83.

9. Alikhan A, Lynch PJ, Eisen DB. Hidradenitis suppurativa: A comprehensive review. J Am Acad Dermatol. 2009;60:539-61.

10. Deckers IE, van der Zee H, Prens EP. Epidemiology of hidradenitis suppurativa: prevalence, pathogenesis and factors associated with the development of HS. Curr Dermatol Rep. 2014:3:54-60.

11. Kohorst JJ, Kimball AB, Davis MD. Systemic associations of hidradenitis suppurativa. J Am Acad Dermatol. 2015;73:S27-S35.

12. Pink AE, Simpson MA, Desai N, Dafou D, Hills A, Mortimer P, et al. Mutations in the gamma-secretase genes NCSTN, PSENEN, and PSEN1 underlie rare forms of hidradenitis suppurativa (acne inversa). J Invest Dermatol. 2012;132:2459-61. 
13. Wang B, Yang W, Wen W, Sun J, Su B, Liu B, et al. Gamma-secretase gene mutations in familial acne inversa. Science. 2010;330: 1065.

14. Pink AE, Simpson MA, Desai N, Trembath RC, Barker JN. Gamma-secretase mutations in hidradenitis suppurativa: new insights into disease pathogenesis. J Invest Dermatol. 2013;133:601-7.

15. Nikolakis G, Join-Lambert O, Karagiannidis I, Guet-Revillet $\mathrm{H}$, Zouboulis CC, Nassif A. Bacteriology of hidradenitis suppurativa/acne inversa: A review. J Am Acad Dermatol. 2015;73:S12-8

16. Revuz JE, Canoui-Poitrine F, Wolkenstein P, Viallette C, Gabison G, Pouget F, et al. Prevalence and factors associated with hidradenitis suppurativa: results from two case-control studies. J Am Acad Dermatol. 2008;59:596-601.

17. van der Zee HH, Laman JD, Boer J, Prens EP. Hidradenitis suppurativa: viewpoint on clinical phenotyping, pathogenesis and novel treatments. Exp Dermatol. 2012;21:735-9.

18. Hutcherson JA, Scott DA, Bagaitkar J. Scratching the surface - tobacco-induced bacterial biofilms. Tob Induc Dis. 2015;13:1.

19. Schlapbach C, Yawalkar N, Hunger RE. Human $\beta$-defensin-2 and psoriasin are overexpressed in lesions of acne inversa. J Am Acad Dermatol. 2009;61:58-65.

20. Wolk K, Warszawska K, Hoeflich C, Witte E, Schneider-Burrus S, Witte K, et al. Deficiency of II-22 contributes to a chronic inflammatory disease: pathogenic mechanisms in acne inversa. J Immunol. 2011;186:1228-39.

21. Miller IM, Rytgaard H, Mogensen UB, Miller E, Ring HC, Ellervik $C$, et al. Body composition and basal metabolic rate in Hidradenitis Suppurativa: a Danish population-based and hospital-based cross-sectional study. J Eur Acad Dermatol Venereol. 2016;30:980-8.

22. van der Zee HH, Jemec GB. New insights into the diagnosis of hidradenitis suppurativa: Clinical presentations and phenotypes. J Am Acad Dermatol. 2015;73:S23-S6.

23. Hurley H. Axillary hyperhidrosis, apocrine bromhidrosis, hidradenitis suppurativa, and familial benign pemphigus: surgical approach. In: Roenigh RR, editor. Dermatologic Surgery. New York: Marcel Dekker;1989.p. 729-39.

24. Danby WF, Hazen PG, Boer J. New and traditional surgical approaches to hidradenitis suppurativa. J Am Acad Dermatol. 2015;73:S62-5.

25.Boer J, Jemec, GB. Resorcinol peels as a possible self-treatment of painful nodules in hidradenitis suppurativa. Clin Exp Dermatol. 2010;35:36-40.

26. Gener G, Canoui-Poitrine F, Revuz JE, Faye O, Poli F, Gabison $\mathrm{G}$, et al. Combination therapy with clindamycin and rifampicin for hidradenitis suppurativa: a series of 116 consecutive patients. Dermatology. 2009;219:148-54.

27. van der Zee HH, Boer J, Prens EP, Jemec GB. The effect of combined treatment with oral clindamycin and oral rifampicin in patients with hidradenitis suppurativa. Dermatology. 2009;219:143-7.

28. Jemec GB, Revuz J, Leyden J. Hidradenitis suppurativa. Berlin: Springer; 2006.

29. Boer J, Nazary M. Long-term results of acitretin therapy for hidradenitis suppurativa. Is acne inversa also a misnomer? Br J Dermatol. 2011;164:170-5.
30.Blok JL van Hattem S, Jonkman MF, Horváth B. Systemic therapy with immunosuppressive agents and retinoids in hidradenitis suppurativa: a systematic review. Br J Dermatol. 2012;168:243-52.

31. Alhusayen R, Shear N. Scientific evidence for the use of current traditional systemic therapies in patients with hidradenitis suppurativa. J Am Acad Dermatol. 2015;73:S42-6.

32. Lee RA, Eisen DB. Treatment of hidradenitis suppurativa with biologic medications. J Am Acad Dermatol. 2015;73:S82-8.

33. Haslund P, Lee RA, Jemec GB. Treatment of hidradenitis suppurativa with tumour necrosis factor alpha inhibitors. Acta Derm Venereol. 2009;89:595-600.

34. Miller I, Lynggaard CD, Lophaven S, Zachariae C, Dufour DN, Jemec GB. A double blind placebo controlled randomised trial of adalimumab in the treatment of hidradenitis suppurativa. Br J Dermatol. 2011;165:391-8.

35. Amano M, Grant A, Kerdel FA. A prospective open-label clinical trial of adalimumab for the treatment of hidradenitis suppurativa. Int J Dermatol. 2010;49:950-5.

36. Van Der Zee HH, de Ruiter L, Van Den Broecke DG, Dik WA, Laman JD, Prens EP. Elevated levels of tumour necrosis factor (TNF)- $\alpha$, interleukin (IL)-1 $\beta$ and IL-10 in hidradenitis suppurativa skin: A rationale for targeting TNF- $\alpha$ and IL-1 $\beta$. Br J Dermatol. 2011;164:1292-8.

37. Grant A, Gonzalez T, Montgomery MO, Cardenas V, Kerdel FA. Infliximab therapy for patients with moderate to severe hidradenitis suppurativa: a randomized, double-blind, placebo-controlled crossover trial. J Am Acad Dermatol. 2010;62:205-17.

38. Giamarellos-Bourboulis EJ, Pelekanou E, Antonopoulou A, Petropoulou H, Baziaka F, Karagianni V, et al. An open-label phase II study of the safety and effi cacy of etanercept for the therapy of hidradenitis suppurativa. Br J Dermatol. 2008;158:567-72.

39. Lee RA, Dommasch E, Treat J, Sciacca-Kirby J, Chachkin S, Williams J, et al. A prospective clinical trial of open-label etanercept for the treatment of hidradenitis suppurativa. J Am Acad Dermatol. 2009;60:565-73.

40. Gulliver WP, Jemec GB, Baker KA. Experience with ustekinumab for the treatment of moderate to severe hidradenitis suppurativa. J Eur Acad Dermatol Venereol. 2012;26:911-4.

41. Sharon VR, Garcia MS, Bagheri S, Goodarzi H, Yang C, Ono $Y$, et al. Management of recalcitrant hidradenitis suppurativa with ustekinumab. Acta Derm Venereol. 2012;92:320-1.

42. Zarchi K, Dufour DN, Jemec GB. Successful treatment of severe hidradenitis suppurativa with anakinra. JAMA Dermatol. 2013;149:1192-4.

43. Leslie KS, Tripathi SV, Nguyen TV, Pauli M, Rosenblum MD. An open-label study of anakinra for the treatment ofmoderate to severe hidradenitis suppurativa. J Am Acad Dermatol. 2014;70:243-51.

44. Madan V, Hindle E, Hussain W, August PJ. Outcomes of treatment of nine cases of recalcitrant severe hidradenitis suppurativa with carbon dioxide laser. Br J Dermatol. 2008;159:1309-14.

45. Hamzavi IH, Griffith JL, Riyaz F, Hessam S, Bechara FG. Laser and light-based treatment options for hidradenitis suppurativa. J Am Acad Dermatol. 2015;73:S78-81. 